\title{
Intrahepatic Injection of Recombinant Adeno-Associated Virus Serotype 2 Overcomes Gender-Related Differences in Liver Transduction
}

\author{
PEDRO BERRAONDO, ${ }^{1,2}$ JULIEN CRETTAZ, ${ }^{1}$ LAURA OCHOA, ${ }^{1}$ ASTRID PAÑEDA, ${ }^{1}$ JESÚS PRIETO,${ }^{1}$ \\ IÑAKI F. TROCÓNIZ, ${ }^{3}$ and GLORIA GONZÁLEZ-ASEGUINOLAZA ${ }^{1}$
}

\begin{abstract}
The liver is an attractive organ for gene therapy because of its important role in many inherited and acquired diseases. Recombinant adeno-associated viruses (rAAVs) have been shown to be good candidates for liver gene delivery, leading to long-term gene expression. We evaluated the influence of the route of administration on rAAV-mediated liver transduction by comparing levels of luciferase expression in the livers of male and female mice after injection of rAAV serotype 2, using three different routes of administration: intravenous (IV), intraportal (IP), or direct intrahepatic (IH) injection. To determine transgene expression we used a noninvasive optical bioluminescence imaging system that allowed long-term in vivo analysis. After IV injection dramatic differences in liver transgene expression were observed, depending on gender. When IP injection was used the differences were reduced although they were still significant. Interestingly, direct intrahepatic injection of rAAV vectors was associated with the fastest and strongest onset of luciferase expression. Moreover, no gender differences in liver transduction were observed and luciferase expression was confined to the site of injection. Thus, direct intrahepatic injection of rAAV offers specific advantages, which support the potential of this route of administration for future clinical applications.
\end{abstract}

\section{INTRODUCTION}

A DENO-ASSOCIATED VIRUSES are small, single-stranded DNA viruses derived from a replication-deficient member of the parvovirus family and do not appear to cause any disease in humans (Lai et al., 2002). Recombinant adeno-associated vectors (rAAVs) are safe and effective gene therapy vectors. They are not pathogenic, do not elicit significant immune responses, transduce both quiescent and dividing cells, and can provide high-level and long-term transgene expression (Lai et al., 2002; Bessis et al., 2004; Buning et al., 2004). Furthermore, it has been described that rAAV integration is inefficient and the persistence of rAAV genomes in tissues is largely due to episomal genomes (Nakai et al., 2001; McCarty et al., 2004). Many of their biological properties make AAV an attractive gene transfer system for clinical application.
AAV particles enter the cell via receptor-mediated endocytosis through clathrin-coated pits. Inside the cells and after endosomal acidification, the virus is released into the cytosol. Upon release into the cytosol, it accumulates perinuclearly and slowly penetrates into the nucleus. Once in the nucleus, the transfection efficiency is further limited by the need to convert the single-stranded (ss) genome into double-stranded (ds), transcriptionally active forms (Bartlett et al., 2000). Depending on the cell type, dsDNA conversion occurs by de novo synthesis of the second strand or through annealing of complementary plus and minus single-stranded molecules (Nakai et al., 2000).

Recombinant AAVs show some degree of liver specificity after systemic injection (Summerford et al., 1999), and have been proved to be highly effective vehicles for gene therapy in several disorders such as glycogen storage disease type II (Sun et al., 2005), hemophilia (High, 2002; Wang et al., 2005),

${ }^{1}$ Laboratory of Gene Therapy of Viral Hepatitis, Division of Gene Therapy and Hepatology, Center for Applied Medical Research (CIMA), University of Navarra, 31080 Pamplona, Navarra, Spain.

${ }^{2}$ Present adress: Régulation Immunitaire et Vaccinologie, Institut Pasteur, 75724 Paris, France.

${ }^{3}$ Department of Pharmacy, School of Pharmacy, University of Navarra, 31008 Pamplona, Navarra, Spain.

P.B. and J.C. contributed equally to this work. 
phenylketonuria (PKU) (Mochizuki et al., 2004), and hypercholesterolemia (Lebherz et al., 2004). They have been successfully used to transduce factor IX to mouse, dog, and nonhuman primate livers and have achieved persistent, curative concentrations of the functionally active molecule (Snyder et al., 1997; Summerford et al., 1999; Harding et al., 2004; Wang et al., 2005). rAAV vectors are currently being evaluated in patients with hemophilia (High, 2004). The routes of administration for rAAV most frequently used to transduce the liver are intravenous injection and portal vein infusion (Harding et al., 2004). AAV administration by hepatic arterial infusion resulted in highly efficient liver transduction (Ohashi et al., 2005). In mice, injection into the splenic capsule has been used to efficiently deliver the AAV vector to the portal circulation (Daly, 2004). Direct intrahepatic injection has been used in fetuses (Mitchell et al., 2000) and in adult female mice (Snyder et al., 1997). Intrahepatic injection of an AAV expressing factor IX resulted in high levels of protein expression; however, liver transduction by this route has not been characterized (Snyder et al., 1997).

Striking differences in liver transduction have been reported after portal vein injection of rAAV serotypes 2 and 5, depending on the sex of the animal (Davidoff et al., 2003). Furthermore, in a mouse model of PKU, about three times more rAAV must be injected into female mice than into male mice to achieve an equivalent reduction in serum phenylalanine (Mochizuki et $a l .$, 2004). These data have been further corroborated by our results in woodchucks chronically infected with the woodchuck hepatitis virus, in which the intraportal administration of rAAV carrying the woodchuck interferon- $\alpha$ (IFN- $\alpha$ ) gene resulted in dramatic differences in cytokine expression depending on the sex (Berraondo et al., 2005). This would seem to indicate that sex differences in AAV-mediated liver transduction found in mice are not species specific and may represent a problem in clinical applications. Analysis of transgene expression in the rodent brain after AAV injection into the thalamus indicates that sex also influences AAV transduction efficiency in this organ (Dodge et al., 2005).

The number of hepatocytes that are stably transduced, even when high doses of rAAV vector are delivered, is relatively low (Nakai et al., 2002; Sanlioglu et al., 2004; Zhong et al., 2004). This might represent a problem when a large animal model is used or when a gene therapy protocol is to be applied in a clinical trial, because of the large amounts of recombinant vector that would have to be administered. Therefore, we investigated whether intrahepatic injection of rAAV might be superior to other injection methods for safe and effective transduction of the liver. Three different routes of administration targeting the liver were used: intrahepatic (IH), intraportal (IP), and intravenous (IV, via the tail vein) injection. Levels of luciferase expression were then compared. The use of a noninvasive optical bioluminescence imaging system allowed long-term quantitative evaluation of transgene expression in living animals as well as the study of vector biodistribution. During these analyses we observed significant differences in AAV-mediated liver transgene expression depending on the route of administration and the gender of the animal. Direct intrahepatic injection of the virus resulted in faster, stronger, and localized liver transduction and led to transgene expression levels in females comparable to those in males injected by the IV or IP route.

\section{MATERIALS AND METHODS}

\section{rAAV vector construction}

Construction of AAV-Luc was performed as described (Berraondo et al., 2005). Briefly, a fragment containing the luciferase cDNA was obtained by polymerase chain reaction (PCR) from plasmid pCDNA2.1-Luc. Plasmid pGTC-wIFNWRE was digested with EcoRI to obtain the expression cassette without wIFN and was treated with T4 DNA polymerase to create blunt ends. Both fragments were ligated to obtain the plasmid pGTC-Luc-WRE. The expression cassette from this plasmid was obtained by digestion with SpeI and SalI and cloned into the plasmid pAAV-Luc. This plasmid was digested with $X b a \mathrm{I}$ and $S a l \mathrm{I}$ and both fragments were ligated. This plasmid was transformed into Escherichia coli strain SURE (Stratagene, La Jolla, CA) and the integrity of viral inverted terminal repeats (ITRs) was confirmed by digestion with $P v u I I, S m a I$, and SnaBI.

\section{rAAV vector production and purification}

Plasmid with the expression cassette flanked with ITRs and helper plasmid pDG (Zhong et al., 2004) were prepared with an EndoFree plasmid mega kit (Qiagen, Valencia, CA). A mixture of plasmid ( $20 \mu \mathrm{g}$ of AAV plasmid and $55 \mu \mathrm{g}$ of pDG per plate) was transfected into $293 \mathrm{~T}$ cells, using linear polyethylenimine (25 kDa; Polysciences, Warrington, PA) as described in Durocher et al. (2002). After $48 \mathrm{hr}$ cells were harvested, resuspended in Dulbecco's modified Eagle's medium (DMEM; $2.5 \mathrm{ml} /$ plate), and lysed by two freeze-thaw cycles. Virus was digested with $0.1 \mathrm{mg}$ each of DNase and RNase (both from Roche Diagnostics, Mannheim, Germany) for $30 \mathrm{~min}$ at $37^{\circ} \mathrm{C}$ and centrifuged for $15 \mathrm{~min}$ at $4^{\circ} \mathrm{C}$ at $3000 \mathrm{rpm}$. The supernatant was treated with $0.5 \%$ deoxycholic acid (Sigma, St. Louis, MO) for $30 \mathrm{~min}$ at $37^{\circ} \mathrm{C}$. It was then clarified by filtration through a $0.22-\mu \mathrm{m}$ filter and the virus was loaded into a HiTrap heparin column (Amersham Biosciences, Uppsala, Sweden) that had been equilibrated with phosphate-buffered saline (PBS, pH 7.4; GIBCO, Paisley, UK) at $1 \mathrm{ml} / \mathrm{min}$. The matrix was washed with $25 \mathrm{ml}$ of PBS- $0.1 \mathrm{M} \mathrm{NaCl}$ and virus was eluted with $15 \mathrm{ml}$ of PBS with $0.4 \mathrm{M} \mathrm{NaCl}$. To exchange the buffer and to concentrate the virus, it was applied to an ULTRAFREE-15 centrifugal filter device (Millipore, Bedford, MA), using PBS-MK (PBS containing $1 \mathrm{mM} \mathrm{MgCl}_{2}$ and $2.5 \mathrm{mM} \mathrm{KCl})$.

For titration an aliquot was digested with DNase and treated with proteinase $\mathrm{K}$ (Roche Diagnostics). After inactivation, the titer was determined by real-time PCR. Primers and TaqMan probes (Applied Biosystems, Foster City, CA) were designed to amplify a fragment of the luciferase gene, using Primer Express software (Applied Biosystems). Primers and probe were as follows: sense, 5' -AACATAAAGAAAGGCCCGGC-3'; antisense, 5'-GCCTTATGCAGTTGCTCTCCA-3'; probe, 5'CATTCTATCCGCTGGAAGATGGAACCG-3' .

The reaction was performed in $20 \mu \mathrm{l}$ with $2 \mu \mathrm{l}$ of sample, $2 \mu \mathrm{l}$ of reaction mix (LightCycler FastStart DNA Master hybridization probes; Roche Diagnostics, Basel, Switzerland), $3.2 \mu \mathrm{l}$ of $\mathrm{MgCl}_{2}(25 \mathrm{mM}), 0.6 \mu \mathrm{l}$ of each primer $(10 \mathrm{mM})$, and $0.4 \mu \mathrm{l}$ of probe $(5 \mathrm{mM})$. PCR was performed according to the following parameters: $10 \mathrm{~min}$ at $95^{\circ} \mathrm{C}$ (denaturation) and $40 \mathrm{cy}$ - 
cles of $0.1 \mathrm{sec}$ at $95^{\circ} \mathrm{C}$ and $20 \mathrm{sec}$ at $60^{\circ} \mathrm{C}$ (hybridization/elongation). The fluorescence signal delivered during PCR amplification was monitored with the LightCycler system (Roche Diagnostics). The titer was expressed as viral genomes (VG) per milliliter.

\section{Mice and $A A V$ administration}

BALB/c or C57BL/6 mice were obtained from Harlan (Barcelona, Spain), housed under specific pathogen-free conditions, and handled according to the guidelines of our institution (Centro de Investigación Farmacobiológica Aplicada, Pamplona, Spain).
AAV administration was performed in 8-week-old mice. For intrahepatic and intraportal injection, mice were anaesthetized with ketamine-xylazine and a partial laparotomy was performed. Mice received a direct injection of $50 \mu \mathrm{l}$ of rAAV virus into the left medial lobe of the liver or into the spleen. Intravenous injection was performed by injecting a total volume of $200 \mu \mathrm{l}$ through the tail vein.

\section{Bioluminescence measurement in living mice}

Mice were anesthetized with ketamine-xylazine and $100 \mu \mathrm{l}$ of D-luciferin (Xenogen, Alameda, CA) at a concentration of $30 \mathrm{mg} / \mathrm{ml}$, diluted in $150 \mathrm{mM} \mathrm{NaCl}$ solution, was injected by
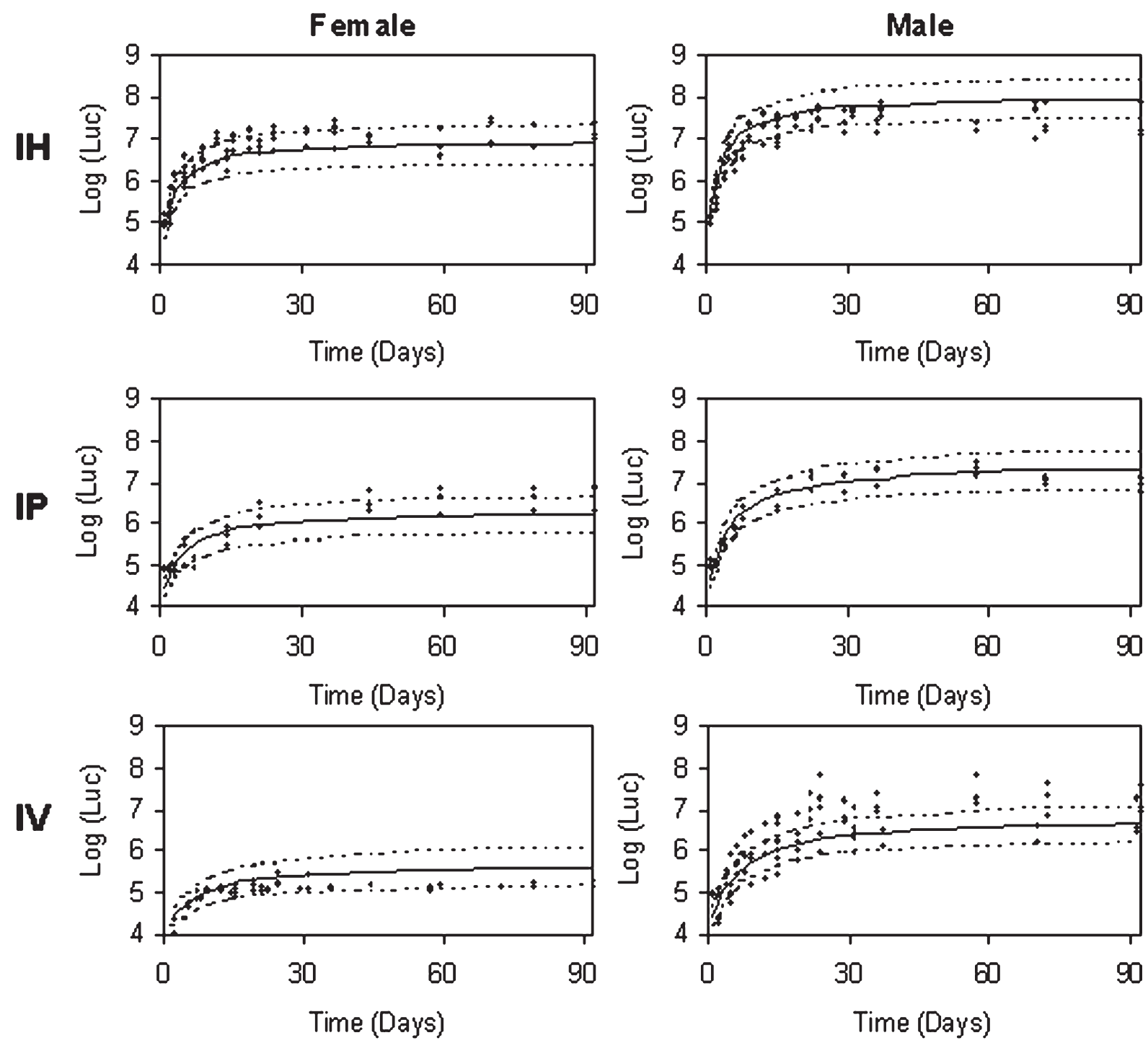

FIG. 1. Short-term in vivo CCD signal intensity in the liver of BALB/c mice injected with low-dose AAV-Luc. Male and female BALB/c mice were injected with AAV serotype 2 expressing luciferase gene, at a dose of $2.5 \times 10^{10}$ genome-containing particles. AAV-Luc was injected intravenously (IV; $n=8$ males and 8 females), intraportally (IP; $n=3$ males and 3 females), or intrahepatically ( $\mathrm{IH} ; n=8$ males and 8 females). Subsequent imaging was performed for luciferase expression, using D-luciferin injected intraperitoneally $(150 \mathrm{mg} / \mathrm{kg})$ and starting on day 1 and every week thereafter. Total bioluminescence was quantified and plotted over time. Each dot represents the decimal logarithm of bioluminescence for each animal at a time point. A solid line shows the mean value of each group; dashed lines indicate the confidence interval. 
Table 1. $L_{\max }$ And $T_{50}$ Values from BALB/c Mice Transfer with $2.5 \times 10^{10}$ VG of AAV-Luc

\begin{tabular}{|c|c|c|c|c|c|c|}
\hline & \multicolumn{3}{|c|}{ Female } & \multicolumn{3}{|c|}{ Male } \\
\hline & $I V[M(S D)]$ & $I P[M(S D)]$ & $I H[M(S D)]$ & $I V[M(S D)]$ & $I P[M(S D)]$ & $I H[M(S D)]$ \\
\hline$L_{\max }$ & $1.46(0.13)$ & $2.64(0.32)$ & $3.26(0.23)$ & $3.21(0.53)$ & $3.38(0.13)$ & $3.80(0.24)$ \\
\hline$T_{50}$ (days) & $5.92(0.03)$ & $4.35(0.13)$ & $2.39(0.24)$ & $5.95(0.18)$ & $4.14(0.10)$ & $2.35(0.21)$ \\
\hline
\end{tabular}

Abbreviations: $L_{\max }$, maximum increase in bioluminescence from baseline; $\mathrm{M}$, mean value; $\mathrm{SD}$, standard deviation; $T_{50}$, time necessary to reach the bioluminescence value $L_{\max } / 2$.

the intraperitoneal route. The animals were placed in the imaging chamber of the Xenogen IVIS system (Xenogen), which includes a cooled charge-coupled device (CCD) camera. A grayscale photograph of the animals was acquired, followed by bioluminescence acquisition. Regions of interest (ROIs) were drawn over the positions of greatest signal intensity on each animal, as well as over regions of "no" signal, which were uses as background readings. Light intensity was quantified as photons $/ \mathrm{sec} / \mathrm{cm}^{2} / \mathrm{sr}$. The gray-scale photograph and data images from all studies were superimposed, using Living Image (Xenogen).

For the ex vivo analysis of luciferase expression, animals were killed on day 92 after AAV-Luc injection and various organs were collected and frozen in liquid nitrogen. The organs collected were as follows: testicles or ovaries, spleen, stomach, lungs, heart, kidney, and liver (separated into left medial lobe, right medial lobe, and right lateral lobe). Tissue samples were homogenized in $1 \times$ lysis buffer and the protocol to detect luciferase in cells (Promega, Madison, WI) was followed.

\section{Data analysis}

All data were analyzed with nonlinear mixed effect models, using MONOLIX software (http://www.math.u-psud.fr/ lavielle/monolix/). Selection between models was based on the precision of the estimated parameters, goodness-of-fit plots, and the value of the Akaike information criterion (AIC). The model with the lowest AIC value was selected, because it indicates that precision of model parameters and data description was adequate (Ludden et al., 1994).
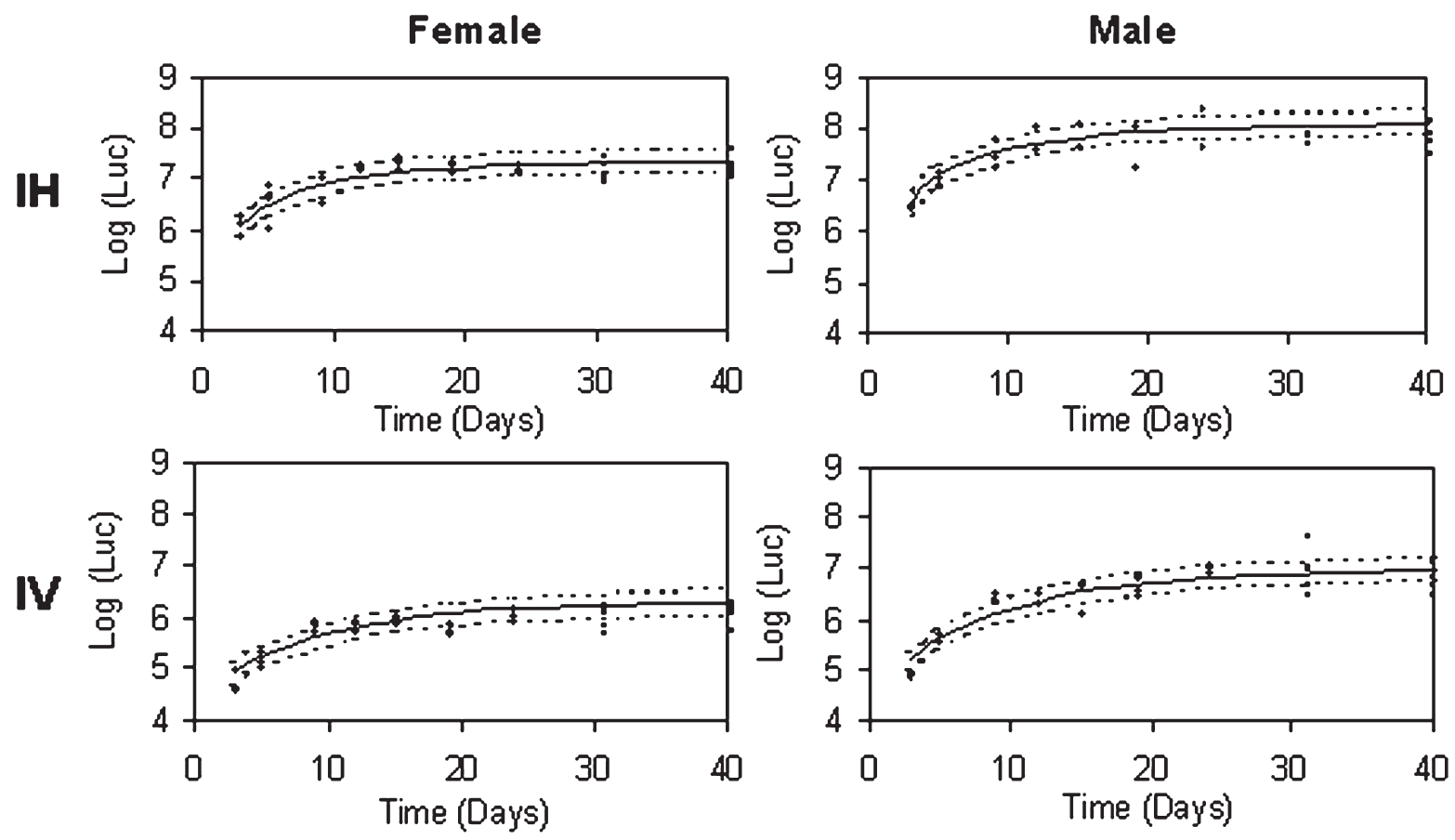

FIG. 2. Short-term in vivo CCD signal intensity in the liver of BALB/c mice injected with a high dose of AAV-Luc. Male and female BALB/c mice were injected with AAV serotype 2 expressing luciferase gene, at a dose of $2.5 \times 10^{11}$ genome-containing particles. AAV-Luc was injected intravenously (IV; $n=5$ males and 5 females) or intrahepatically (IH; $n=5$ males and 5 females). Subsequent imaging was performed for luciferase expression, using D-luciferin injected intraperitoneally $(150 \mathrm{mg} / \mathrm{kg})$ and starting on day 3 and every week thereafter. Total bioluminescence was quantified and plotted over time. Each dot represents the decimal logarithm of bioluminescence for each animal at a time point. A solid line shows the mean value of each group; dashed lines indicate the confidence interval. 
Table 2. $L_{\max }$ And $T_{50}$ VAlues from BALB/C Mice Transfer WITH $25 \times 10^{11}$ VG OF AAV-LUC

\begin{tabular}{|c|c|c|c|c|}
\hline & \multicolumn{2}{|c|}{ Female } & \multicolumn{2}{|c|}{ Male } \\
\hline & $I V[M(S D)]$ & $I H[M(S D)]$ & $I V[M(S D)]$ & $I H[M(S D)]$ \\
\hline$L_{\max }$ & $2.64(0.036)$ & $3.63(0.062)$ & $3.56(0.034)$ & $4.32(0.183)$ \\
\hline$T_{50}$ (days) & $5.83(0.00020)$ & $2.15(0.00062)$ & $5.83(0.0020)$ & $2.15(0.00015)$ \\
\hline
\end{tabular}

Abbreviations: $L_{\max }$, maximum increase of bioluminescence from baseline; M, Mean value; SD, standard deviation $T_{50}$, time necessary to reach the bioluminescence value $L_{\max } / 2$.

Because of the large range in luciferase expression, data were first subjected to logarithmic transformation before analysis. The logarithmic value of the luciferase expression $(L)$ after AAV injection was modeled by assuming a saturable absorption process and a negligible elimination process in the time frame considered. The background level of bioluminescence (nonspecific light) was determined by acquiring the light of mice that had not been injected with luciferin, and was found to be equal to 4 .

$$
L=4+\frac{L_{\max } \cdot t}{T_{50}+t}
$$

where $L_{\max }$ represents the maximum increase in bioluminescence from baseline, $T_{50}$ represents the time necessary to reach the bioluminescence value $L_{\max } / 2$, and $t$ represents time.

Statistical analysis of the various estimated individual parameters was performed by two-way analysis of variance (ANOVA) followed by multiple comparison, using the least significant difference test (SPSS software; SPSS, Chicago, IL).

The AAV half-life in liver is determined by fitting the longterm follow-up data to an elimination process, assuming that the absorption process in this time frame is negligible:

$$
L=L_{\mathrm{basal}} \cdot e^{-k_{e} t}
$$

where $L_{\text {basal }}$ represents the bioluminescence value at steady state and $k_{\mathrm{e}}$ is the constant of the elimination process. The elimina-
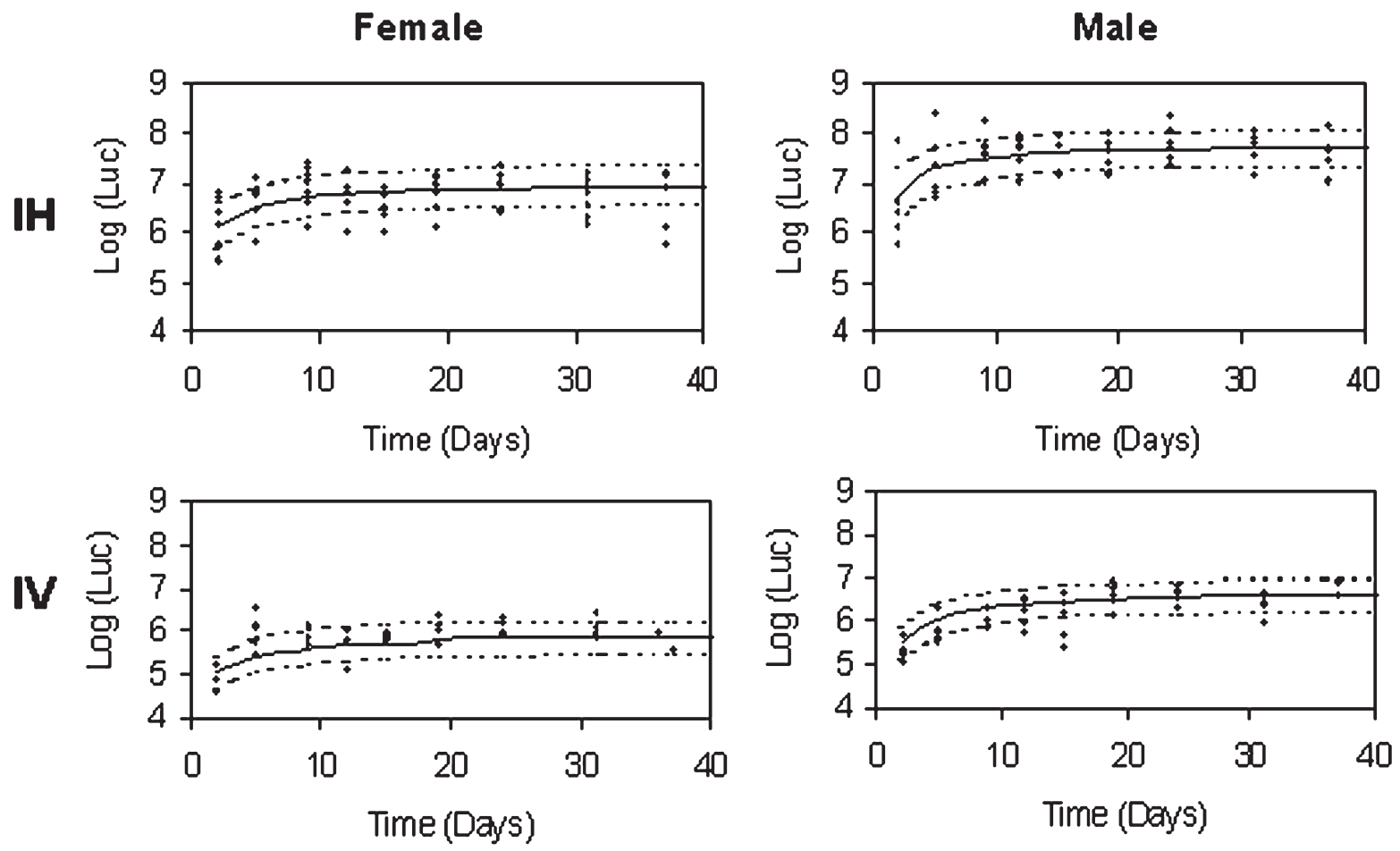

FIG. 3. Short-term in vivo CCD signal intensity in the liver of C57BL/6 mice injected with low-dose AAV-Luc. Male and female C57BL/6 mice were injected with AAV serotype 2 expressing luciferase gene, at a dose of $2.5 \times 10^{10}$ genome-containing particles. AAV-Luc was injected intravenously (IV; $n=5$ males and 5 females) or intrahepatically (IH; $n=6$ males and 7 females). Subsequent imaging was performed for luciferase expression, using D-luciferin injected intraperitoneally (150 mg/kg) and starting on day 1 and every week thereafter. Total bioluminescence was quantified and plotted over time. Each dot represents the decimal logarithm of bioluminescence for each animal at a time point. A solid line shows the mean value of each group; dashed lines indicate the confidence interval. 
Table 3. $L_{\max }$ And $T_{50}$ Values from C57BL/6 Mice Transfer with $2.5 \times 10^{10} \mathrm{VG}$ of AAV-Luc

\begin{tabular}{llllll}
\hline & \multicolumn{2}{c}{ Female } & & \multicolumn{2}{c}{ Male } \\
\cline { 2 - 3 } & $I V[M(S D)]$ & $I H[M(S D)]$ & & $I V[M(S D)]$ & $I H[M(S D)]$ \\
\hline$L_{\max }$ & $2.05(0.089)$ & $2.93(0.184)$ & & $2.64(0.135)$ & $3.88(0.212)$ \\
$T_{50}$ (days) & $1.64(0.121)$ & $0.79(0.359)$ & & $1.90(0.095)$ & $0.97(0.445)$ \\
\hline
\end{tabular}

Abbreviations: $L_{\max }$, maximum increase in bioluminescence from baseline; M, mean value; $\mathrm{SD}$, standard deviation; $T_{50}$, time necessary to reach the bioluminescence value $L_{\text {max }} / 2$.

tion half-life obtained from the estimated individual parameter $k_{\mathrm{e}}$ was compared by Kruskall-Wallis test.

\section{RESULTS}

In vivo quantitative analysis of luciferase expression after AAV-Luc injection in liver

Male and female BALB/c mice were injected with a single dose of AAV-Luc at $2.5 \times 10^{10} \mathrm{VG}$ using three different routes of administration: intravenous (IV; $n=8$ ), intraportal (IP; $n=$ 3 ), and direct injection into the liver (intrahepatic $[\mathrm{IH}] ; n=8$ ). Whole body imaging was performed from day 1 postinjection up to day 92 postinjection and luciferase expression was quantified. Bioluminescence data were measured as photons/sec/ $\mathrm{cm}^{2} / \mathrm{sr}$ and analyzed in a pharmacokinetic model in which the "drug" is the transcriptionally active double-stranded DNA intermediate of the recombinant AAV. The effect of our drug is measured and represented as the logarithm of the bioluminescence value. This parameter is assumed to be proportional to the quantity of the drug. Quantitative data were fitted to a model of a saturable absorption process to perform a detailed statistical analysis (Fig. 1). In this model, we have defined $L_{\max }$ as the maximum increase in bioluminescence from baseline and $T_{50}$ as the time necessary to reach the bioluminescence value $L_{\max } / 2$.

The results are summarized in Table 1 . Analysis of $L_{\max }$ and $T_{50}$ values from all the animals was performed with nonlinear mixed effect models. Model development was driven by the data; the value of the $L_{\max }$ parameter was affected by both gender and route of administration, whereas $T_{50}$ was affected only by the route of administration. Statistical analysis of $L_{\max }$ and $T_{50}$, using the least significant difference (LSD) test with a significance level of 0.05 , showed that on the basis of $L_{\max }$ four groups of animals showed significant differences: (1) IV female mice, showing the lowest value; (2) IP female mice and (3) IH female mice and IP and IV male mice, showing lower and higher intermediate values, respectively; and (4) IH male mice, showing the highest transgene expression values (Table 1).

The $T_{50}$ value was affected by route, but was independent of gender. Therefore, three different subsets of animals could be distinguished: (1) IV mice, the subset requiring the longest time to reach this value; (2) IP mice, the intermediate subset; (3) IH mice, the third subset, expressing the transgene with the fastest kinetics. The most striking observation from the analysis of this parameter is the short period of time required to reach $T_{50}$ after IH injection (Table 1).
Our data indicate that IH injection clearly improves AAVmediated liver transduction in $\mathrm{BALB} / \mathrm{c}$ mice, using a relatively low viral dose. To further confirm our observations we tested the effect of the route of administration, using a higher dose of virus and a different strain of mouse, C57BL/6. In these studies we analyzed IH and IV routes of administration.

Male and female BALB/c mice $(n=5)$ were injected $\mathrm{IH}$ and IV with a single dose of AAV-Luc at $2.5 \times 10^{11}$ VG. Bioluminescence data were measured from day 3 postinjection up to day 40 postinjection and analyzed as previously described. As shown in Fig. 2 and Table 2, the differences in $L_{\max }$ shown by female mice were reduced, depending on the route of administration, when the dose of virus was increased; however, they were still significant (females: $L_{\max } \mathrm{IH}=3.63$ versus $L_{\max }$ $\mathrm{IV}=2.64 ; p>0.05)$. Regarding $T_{50}$, as shown in Table 2 , the values are identical to those obtained when using the low dose, indicating that the kinetics of transgene expression are independent of the dose of virus administered.

To analyze the effect of mouse strain on our results, male and female C57BL/6 mice $(n=5)$ were injected IH and IV with a single dose of AAV-Luc at $2.5 \times 10^{10} \mathrm{VG}$. Bioluminescence data were measured from day 3 postinjection up to day 37 postinjection and analyzed as previously described. As shown in Fig. 3 and Table 3, as previously observed, the lowest expression level was achieved in female mice after IV injection. IH injection resulted in the highest levels of transgene expression in both male and female mice, with the luciferase expression levels of IH female mice becoming equivalent to those of IV male mice. Regarding the $T_{50}$ parameter, the kinetics of transgene expression were faster after IH injection in both groups; interestingly, $T_{50}$ values were smaller than in BALB/c mice after IV and IH injection, indicating faster expression kinetics (Table 3 ).

\section{Comparison of transgene expression when steady state levels were reached}

Luciferase transgene expression increased over time until steady state levels were reached approximately 4 weeks after rAAV administration in all the groups of female mice. Thereafter there was stable expression for more than 17 months (Fig. 4) (length of the study). $L_{\text {basal }}$, which is the bioluminescence value at steady state, and $k_{\mathrm{e}}$, the constant of the elimination process, were calculated for every group (Table 4). The intensity of the signal slowly declined over time, and fitting the data to an exponential elimination model allowed us to determine a median elimination half-life of 31 years for the IV-injected animals, 55 years for the IP-injected animals 


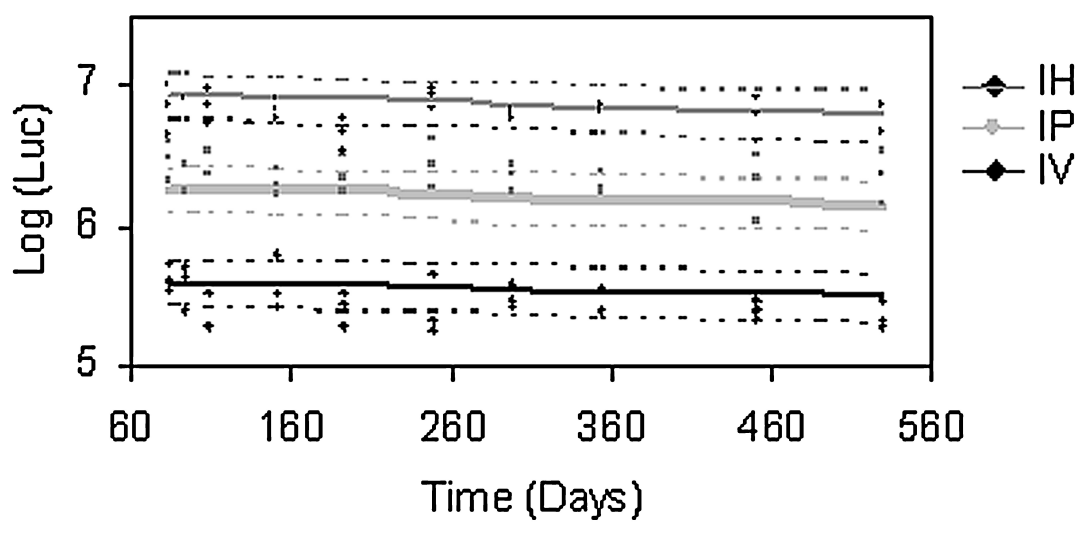

FIG. 4. Long-term in vivo $\mathrm{CCD}$ signal intensity in the liver of BALB/c mice injected with low-dose AAV-Luc. Female BALB/c mice $(n=3)$ were injected with AAV serotype 2 expressing luciferase gene at a dose of $2.5 \times 10^{10}$ genome-containing particles. AAV-Luc was injected IP, IV, or IH. Each dot represents the decimal logarithm of the bioluminescence value for each animal at each time point. A solid line shows the mean value of each group; dashed lines indicate the confidence interval.

FIG. 5. Bioluminescence imaging of AAV-Luc gene expression in living mice, using a cooled CCD camera. Eight-week-old male and female $\mathrm{BALB} / \mathrm{c}$ mice were injected with AAV serotype 2 expressing the luciferase gene, at a dose of $2.5 \times$ $10^{10}$ genome-containing particles and using different routes. The mice were repeatedly scanned with the CCD camera as described in Fig. 2. Optical CCD images for luciferase expression 96 days postinjection, once a steady state level of luciferase expression was reached, are shown. (A) Male and female mice injected intravenously (IV); (B) male and female mice injected intraportally (IP); (C) male and female mice injected intrahepatically (IH).

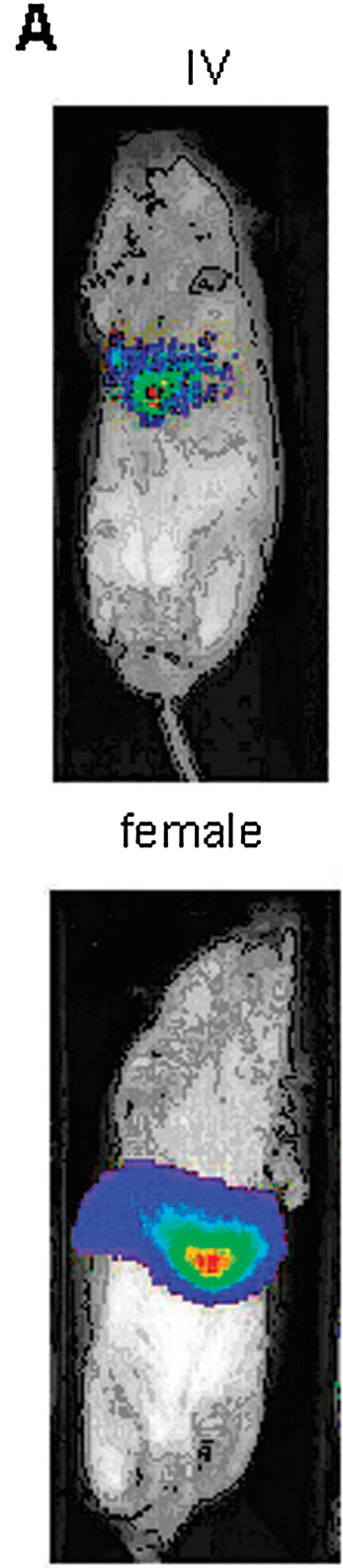

male

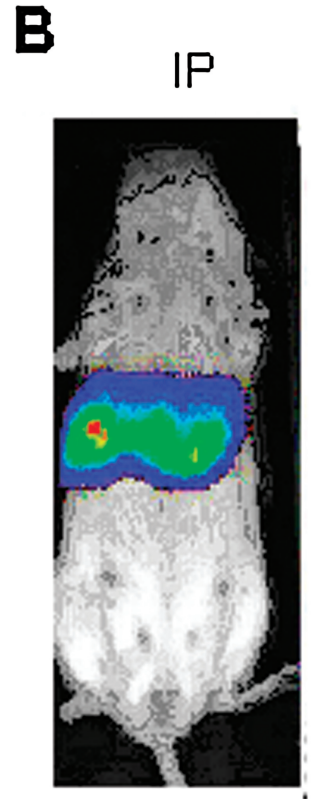

female

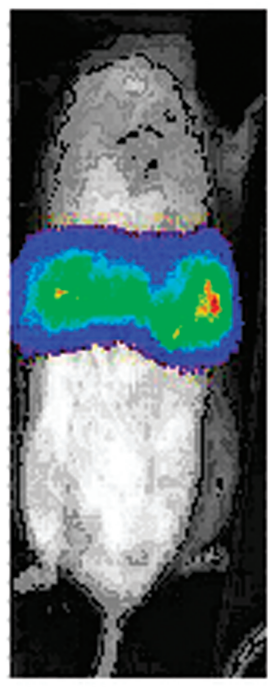

male

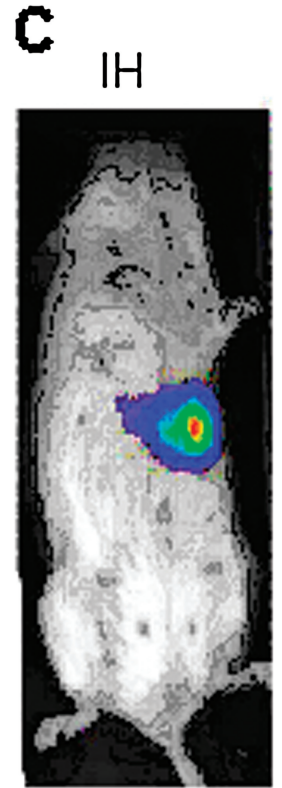

female

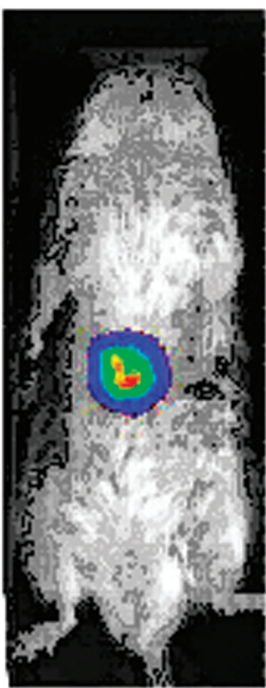

male 
A

IV

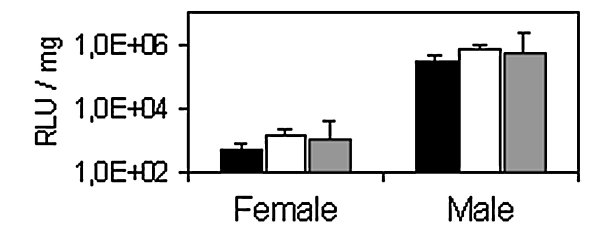

B

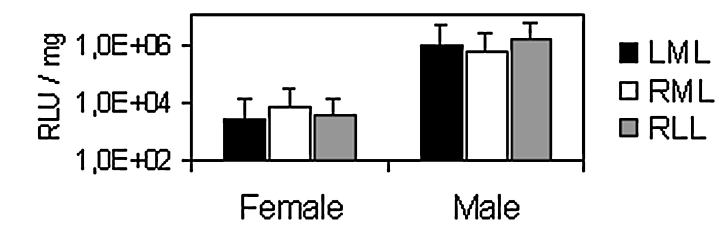

C

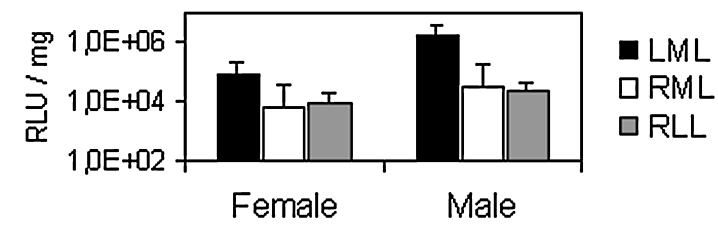

FIG. 6. Ex vivo analysis of luciferase expression in liver lobes. Female and male BALB/c mice were injected with $2.5 \times$ $10^{10}$ genome-containing particles of AAV-Luc by three different routes (IV, IP, and IH). Ninety-two days later, mice were killed and three of the hepatic lobes were removed: the left medial lobe (LML) (injected lobe), the right medial lobe (RML), and the right lateral lobe (RLL). Luciferase expression was tested in the homogenized tissue, using a luminometer. The $y$ axis indicates the median relative light units (RLU) per milligram of protein of each group. (A) IV injected animals; (B) IP injected animals; (C) IH injected animals. After IH injection the injected lobe showed the highest luciferase expression value $(p<0.05$; ANOVA); after IV and IP injection luciferase activity was uniformly distributed throughout the main hepatic lobes.

and 35 years for the IH-injected animals (Table 4), which means life-long expression of the transgene in the liver of all animals after AAV injection. Differences in the half-life value among the groups were not statistically significant, indicating that the elimination process does not depend on the route of administration.

\section{Biodistribution of luciferase expression}

As shown in Fig. 5, by the time luciferase expression reached steady state levels in the liver, no luciferase expression could be detected in other organs independently of the route of administration or gender. Furthermore, expression of luciferase was evenly distributed throughout the entire liver after IV and IP administration of AAV-Luc (Fig. 5A and B), whereas IH administration of the virus resulted in higher expression of luciferase at the site of injection (Fig. 5C).

To confirm and supplement in vivo imaging data, animals from the first study (BALB/c mice injected with $2.5 \times 10^{10} \mathrm{VG}$ of AAV-Luc) were killed 3 months after AAV injection and whole organ preparations were analyzed ex vivo for luciferase activity, using a luminometer. To perform the analysis of luciferase expression, the three major lobes of the liver were excised: left medial lobe (LML), right medial lobe (RML), and right lateral lobe (RLL). We also tested luciferase expression in other organs, such as testicles or ovaries, spleen, stomach, lungs, heart, kidney, and injected muscle. As shown in Fig. 6, ex vivo analysis confirmed the findings based on bioluminescence image analysis. Luciferase expression after IH injection was confined mainly to the injected left medial lobe. Expression in the other lobes was more than 100-fold lower. However, luciferase expression in the liver of IV- and IP-injected mice was evenly distributed. Analysis of luciferase expression in the rest of the organs tested revealed no luciferase expression after IH injection. Low levels of luciferase expression were detected in the spleen and heart of male mice given IV injections (278.60 and 1585.73 RLU/mg of tissue protein, respectively) and in the spleen and kidney of male mice given IP injections (647.71 and $6203.65 \mathrm{RLU} / \mathrm{mg}$ of tissue protein, respectively).

\section{DISCUSSION}

We constructed an rAAV vector that expresses luciferase under the transcriptional control of a constitutive promoter to evaluate in vivo the effect of the route of administration on AAVmediated liver transduction. A detailed and quantitative long-term in vivo follow-up of the biodistribution of transgene expression after the administration of rAAV was performed. The bioluminescence data were analyzed using a pharmacokinetic model in which the "drug" was the transcriptionally active double-stranded DNA intermediate of the recombinant AAV and the "effect" of the drug was measured and represented as the logarithm of the bioluminescence value. This parameter was assumed to be proportional to the quantity of the drug. Quantitative data were fitted to a model of a saturable absorption process to perform a detailed statistical analysis. Our results show that luciferase expression levels in the liver clearly depend on the route of AAV administration and the gender of the animal, whereas the kinetics of AAV-mediated transgene expression were affected by the route of administration and, in-

Table 4. $L_{\text {basal }}$ And $k_{\mathrm{e}}$ Values from BALB/c Female Mice Transfer with $2.5 \times 10^{10} \mathrm{VG}$ of AAV-Luc

\begin{tabular}{lccc}
\hline & $I V[M(S D)]$ & $I P[M(S D)]$ & $I H[M(S D)]$ \\
\hline$L_{\text {basal }}$ & $5.575(0.089)$ & $6.377(0.105)$ & $6.904(0.046)$ \\
$k_{\mathrm{e}}\left(\right.$ years $\left.^{-1}\right)$ & $0.0230(0.020)$ & $0.0081(0.0151)$ & $0.0195(0.070)$ \\
\hline
\end{tabular}

Abbreviations: $L_{\text {basal }}$, bioluminescence value of steady state; $\mathrm{M}$, mean value; $\mathrm{SD}$, standard deviation; $k_{\mathrm{e}}$, constant of the elimination process. 
terestingly, by the strain of mouse and was independent of the sex. All animals demonstrated life-long luciferase expression with no evidence of vector silencing or immune response against the transgene, even though we were using a nonmammalian protein.

As previously described (Davidoff et al., 2003; Berraondo et al., 2005), differences in the magnitude of transgene expression were found in the liver after IV administration of rAAV2, depending on the gender of the animal (Fig. 1). However, we also found that these differences could be minimized by modifying the route of administration. Intraportal injection reduced the difference from 100-fold to less than 10-fold and IH injection further reduced the difference to less than 3-fold. Those results, obtained with a relatively low dose of virus, were reproduced with a 10-fold higher amount (Fig. 2) and were independent of the strain of mouse (Fig. 3). Interestingly, IV and IP injection procedures in male mice resulted in equivalent transgene expression, whereas IH injection resulted in the highest level of transgene expression. Thus IH injection clearly improves liver transduction independently of gender, the dose of virus, or the strain of mouse.

The mechanism underlying the lower efficiency of AAVmediated liver transduction observed in female mice is currently unknown. Davidoff et al. (2003) suggested that the differences were due to an androgen-dependent pathway, associated with the presence of male-specific cellular factors that lead to higher level of rAAV genomes in male than in female mice. The data obtained from our study showed that the differences in gene expression depending on gender were minimized when the virus was administered by liver-directed injection. These results suggest that increasing the local concentration of rAAV in the liver might overcome the lack of certain androgen-related factor(s) or, alternatively, that $\mathrm{IH}$ injection induces the expression of those factors, necessary for the establishment of AAV-mediated transgene expression in females. However, we cannot rule out the possibility that liver-directed injections might bypass the inhibitory effect of factors present in the blood of animals.

Furthermore, the analysis of transgene expression in mice given IH injections showed a faster onset of luciferase expression in comparison with mice given IV and IP injections. The rate-limiting steps of AAV transduction efficacy are the relatively slow nuclear import of AAV genomes, the rate of virus uncoating, and the conversion of ssDNA to transcriptionally competent double-stranded templates (Alexander et al., 1994; Ferrari et al., 1996; Bartlett et al., 2000; Chen et al., 2001; Thomas et al., 2004). In the liver, it has been suggested that dsDNA conversion occurs through annealing of complementary plus and minus single-stranded molecules, not by the de novo synthesis of the second strand; if this is the case the rate of uncoating is a key determinant for the ability of complementary molecules to convert to biologically active molecular forms (Nakai et al., 2000). IH injection might be associated with faster entrance of AAV genomes into the nucleus or with a faster uncoating of vector genomes, which would allow, as in the case of other pseudotyped vectors (Thomas et al., 2004), a more efficient formation of AAV double-stranded templates. However, there is still a certain degree of controversy regarding the mechanism of the formation of transcriptionally active forms and other explanation may be plausible as well (Qing et al., 1998; Miao et al., 2000; Nakai et al., 2000).
The explanation we favor, on the basis of our observations, is that intrahepatic injection first reduces the contact of AAV vectors with inhibitory molecules present in the blood and concentrates the virus in the liver. Once in the liver, the virus undergoes faster uncoating (by a mechanism that requires further studies to be elucidated), resulting in faster formation of transcriptionally active forms.

The in vivo and ex vivo analysis of luciferase expression after intravenous or intraportal AAV administration showed a wide distribution throughout the liver and a low level of expression in some organs in male but not in female mice (data not shown). This might indicate that the gender dependency detected in the liver also occurs in other organs (Dodge et al., 2005). After intrahepatic injection, luciferase expression is restricted mostly to the injected hepatic lobe, with low levels of expression in the other hepatic lobes and nondetectable levels of expression in the rest of the organs analyzed.

In summary, AAV intrahepatic injection offers several advantages over other routes of administration: faster transgene expression, maximum protein expression, and expression confined to the injected hepatic lobe. Finally, direct intrahepatic injection of the virus by ultrasonographic guidance could be easily performed in humans and in large animals. All these factors point to the potential of this route of administration for future clinical applications.

\section{ACKNOWLEDGMENTS}

This work was supported by grants UTE project CIMA, Instituto Salud Carlos IIIC03/02, and SAF 2002-0327 (Ministerio Educación y Ciencia) to J.P., G.G.-A., and P.B. and by the Department of Education and Culture of the Government of Navarra (IIQ4273). The authors thank Africa Vales and Cristina Olagüe for technical assistance and Puri Fortes and Cristian Smerdou for critical reading of the manuscript. Pedro Berraondo was in receipt of grant 009175 from FIS (Ministerio de Salud). Laura Ochoa was in receipt of a grant from Fundación Areces. Astrid Pañeda was in receipt of a grant from Fundación Inocente. Julien Crettaz was a recipient of a grant from Gobierno de Navarra.

\section{REFERENCES}

ALEXANDER, I.E., RUSSELL, D.W., and MILLER, A.D. (1994). DNA-damaging agents greatly increase the transduction of nondividing cells by adeno-associated virus vectors. J. Virol. 68, 8282-8287.

BARTLETT, J.S., WILCHER, R., and SAMULSKI, R.J. (2000). Infectious entry pathway of adeno-associated virus and adeno-associated virus vectors. J. Virol. 74, 2777-2785.

BERRAONDO, P., OCHOA, L., CRETTAZ, J., ROTELLAR, F., VALES, A., MARTINEZ-ANSO, E., ZARATIEGUI, M., RUIZ, J., GONZÁLEZ-ASEGUINOLAZA, G., and PRIETO, J. (2005). IFN- $\alpha$ gene therapy for woodchuck hepatitis with adeno-associated virus: Differences in duration of gene expression and antiviral activity using intraportal or intramuscular routes. Mol. Ther. 12, 68-76. BESSIS, N., GARCIACOZAR, F.J., and BOISSIER, M.C. (2004). Immune responses to gene therapy vectors: Influence on vector function and effector mechanisms. Gene Ther. 11, 10-17. 
BUNING, H., BRAUN-FALCO, M., and HALLEK, M. (2004). Progress in the use of adeno-associated viral vectors for gene therapy. Cells Tissues Organs 177, 139-150.

CHEN, Z.Y., YANT, S.R., HE, C.Y., MEUSE, L., SHEN, S., and KAY, M.A. (2001). Linear DNAs concatemerize in vivo and result in sustained transgene expression in mouse liver. Mol. Ther. 3, 403-410.

DALY, T.M. (2004). AAV-mediated gene transfer to the liver. Methods Mol. Biol. 246, 195-199.

DAVIDOFF, A.M., NG, C.Y., ZHOU, J., SPENCE, Y., and NATHWANI, A.C. (2003). Sex significantly influences transduction of murine liver by recombinant adeno-associated viral vectors through an androgen-dependent pathway. Blood 102, 480-488.

DODGE, J.C., CLARKE, J., PASSINI, M.A., SONG, A., O'RIORDAN, C.R., CHENG, S.H., and STEWART, G.R. (2005). Sex and estrous cycle stage influence the efficiency of AAV-mediated gene transfer in the rodent brain. American Society of Gene Therapy, 8th Annual Meeting. Mol. Ther. 11, S192.

DUROCHER, Y., PERRET, S., and KAMEN, A. (2002). High-level and high-throughput recombinant protein production by transient transfection of suspension-growing human 293-EBNA1 cells. Nucleic Acids Res. 30, E9.

FERRARI, F.K., SAMULSKI, T., SHENK, T., and SAMULSKI, R.J. (1996). Second-strand synthesis is a rate-limiting step for efficient transduction by recombinant adeno-associated virus vectors. J. Virol. 70, 3227-3234.

HARDING, T.C., KOPRIVNIKAR, K.E., TU, G.H., ZAYEK, N., LEW, S., SUBRAMANIAN, A., SIVAKUMARAN, A., FREY, D., HO, K., VANROEY, M.J., NICHOLS, T.C., BELLINGER, D.A., YENDLURI, S., WAUGH, J., McARTHUR, J., VERES, G., and DONAHUE, B.A. (2004). Intravenous administration of an AAV-2 vector for the expression of factor IX in mice and a dog model of hemophilia B. Gene Ther. 11, 204-213.

HIGH, K.A. (2002). AAV-mediated gene transfer for hemophilia. Genet. Med. 4, 56S-61S.

HIGH, K.A. (2004). Clinical gene transfer studies for hemophilia B. Semin. Thromb. Hemost. 30, 257-267.

LAI, C.M., LAI, Y.K., and RAKOCZY, P.E. (2002). Adenovirus and adeno-associated virus vectors. DNA Cell Biol. 21, 895-913.

LEBHERZ, C., GAO, G., LOUBOUTIN, J.P., MILLAR, J., RADER, D., and WILSON, J.M. (2004). Gene therapy with novel adeno-associated virus vectors substantially diminishes atherosclerosis in a murine model of familial hypercholesterolemia. J. Gene Med. 6, 663-672.

LUDDEN, T.M., BEAL, S.L., and SHEINER, L.B. (1994). Comparison of the Akaike Information Criterion, the Schwarz criterion and the $F$ test as guides to model selection. J. Pharmacokinet. Biopharm. 22, 431-445.

McCARTY, D.M., YOUNG, S.M., JR., and SAMULSKI, R.J. (2004). Integration of adeno-associated virus (AAV) and recombinant AAV vectors. Annu. Rev. Genet. 38, 819-845.

MIAO, C.H., NAKAI, H., THOMPSON, A.R., STORM, T.A., CHIU, W., SNYDER, R.O., KAY, M.A. (2000). Nonrandom transduction of recombinant adeno-associated virus vectors in mouse hepatocytes in vivo: Cell cycling does not influence hepatocyte transduction. J. Virol. 74, 3793-3803.

MITCHELL, M., JEREBTSOVA, M., BATSHAW, M.L., NEWMAN, K., and YE, X. (2000). Long-term gene transfer to mouse fetuses with recombinant adenovirus and adeno-associated virus (AAV) vectors. Gene Ther. 7, 1986-1992.

MOCHIZUKI, S., MIZUKAMI, H., OGURA, T., KURE, S., ICHINOHE, A., KOJIMA, K., MATSUBARA, Y., KOBAYAHI, E., OKADA, T., HOSHIKA, A., OZAWA, K., and KUME, A. (2004). Long-term correction of hyperphenylalaninemia by AAV-mediated gene transfer leads to behavioral recovery in phenylketonuria mice. Gene Ther. 11, 1081-1086.

NAKAI, H., YANT, S.R., STORM, T.A., FUESS, S., MEUSE, L., and KAY, M.A. (2001). Extrachromosomal recombinant adeno-associ- ated virus vector genomes are primarily responsible for stable liver transduction in vivo. J. Virol. 75, 6969-6976.

NAKAI, H., THOMAS, C.E., STORM, T.A., FUESS, S., POWELL, S., WRIGHT, J.F., and KAY, M.A. (2002). A limited number of transducible hepatocytes restricts a wide-range linear vector dose response in recombinant adeno-associated virus-mediated liver transduction. J. Virol. 76, 11343-11349.

NAKAI, H.T., STORM, T.A., and KAY, M.A. (2000). Recruitment of single-stranded recombinant adeno-associated virus vector genomes and intermolecular recombination are responsible for stable transduction of liver in vivo. J. Virol. 74, 9451-9463.

OHASHI, K., NAKAI, H., COUTO, L.B., and KAY, M.A. (2005). Modified infusion procedures affect recombinant adeno-associated virus vector type 2 transduction in the liver. Hum. Gene Ther. 16, 299-306.

QING, K., KHUNTIRAT, B., MSH, C., KUBE, D.M, WANG, X.S., PONNAZHAGAN, S., ZHOU, S., DWARKI, V.J., YODER, M.C., and SRIVASTAVA, A. (1998). Adeno-associated virus type 2-mediated gene transfer: Correlation of tyrosine phosphorylation of the cellular single-stranded D sequence-binding protein with transgene expression in human cells in vitro and murine tissues in vivo. J. Virol. 72, 1593-1599.

SANLIOGLU, A.D., KARACAY, B., BENSON, P.K., ENGELHARDT, J.F., and SANLIOGLU, S. (2004). Novel approaches to augment adeno-associated virus type-2 endocytosis and transduction. Virus Res. 104, 51-59.

SNYDER, R.O., MIAO, C.H., PATIJN, G.A., SPRATT, S.K., DANOS, O., NAGY, D., GOWN, A.M., WINTHER, B., MEUSE, L., COHEN, L.K., THOMPSON, A.R., and KAY, M.A. (1997). Persistent and therapeutic concentrations of human factor IX in mice after hepatic gene transfer of recombinant AAV vectors. Nat. Genet. 16, 270-276.

SUMMERFORD, C., BARTLETT, J.S., and SAMULSKI, R.J. (1999). $\mathrm{V}_{\beta} 5$ integrin: A co-receptor for adeno-associated virus type 2 infection. Nat. Med. 5, 78-82.

SUN, B., ZHANG, H., FRANCO, L.M., YOUNG, S.P., SCHNEIDER, A., BIRD, A., AMALFITANO, A., CHEN, Y.T., and KOEBERL, D.D. (2005). Efficacy of an adeno-associated virus 8-pseudotyped vector in glycogen storage disease type II. Mol. Ther. 11, 57-65.

THOMAS, C.E., STORM, T.A., HUANG, Z., and KAY, M.A. (2004). Rapid uncoating of vector genomes is the key to efficient liver transduction with pseudotyped adeno-associated virus vectors. J. Virol. 78, 3110-3122.

WANG, L., CALCEDO, R., NICHOLS, T.C., BELliNGER, D.A., DILLOW, A., VERMA, I.M., and WILSON, J.M. (2005). Sustained correction of disease in naive and AAV2-pretreated hemophilia B dogs: AAV2/8-mediated, liver-directed gene therapy. Blood 105, 3079-3086.

ZHONG, L., LI, W., YANG, Z., CHEN, L., LI, Y., QING, K., WEIGEL-KELlEY, K.A., YODER, M.C., SHOU, W., and SRIVASTAVA, A. (2004). Improved transduction of primary murine hepatocytes by recombinant adeno-associated virus 2 vectors in vivo. Gene Ther. 11, 1165-1169.

Address reprint requests to: Dr. Gloria González-Aseguinolaza Centro de Investigación Médica Aplicada (CIMA) Avenida Pío XII, 55 31080 Pamplona, Navarra, Spain

E-mail: ggasegui@unav.es

Received for publication August 9, 2005; accepted after revision March 10, 2006.

Published online: May 5, 2006. 